\title{
Noninvasive measurement of tissue blood oxygenation with Cerenkov imaging during therapeutic radiation delivery
}

\author{
Xiaofeng Zhang, ${ }^{1, *}$ (1) Sal Kit Lam, ${ }^{2}$ Gregory Palmer, ${ }^{2}$ Shiva Das, ${ }^{3}$ Mark Oldham, ${ }^{2}$ and \\ MARK DeWHIRST ${ }^{2}$ \\ ${ }^{1}$ Department of Radiology, Duke University Medical Center, Durham, North Carolina 27710, USA \\ ${ }^{2}$ Department of Radiation Oncology, Duke University Medical Center, Durham, North Carolina 27710, USA \\ ${ }^{3}$ Department of Radiation Oncology, University of North Carolina, Chapel Hill, North Carolina 27710, USA \\ *Corresponding author: steve.zhang@duke.edu
}

Received 20 June 2017; accepted 11 July 2017; posted 14 July 2017 (Doc. ID 300529); published 8 August 2017

Tumor tissue oxygenation significantly affects the outcome of radiotherapy. Real-time monitoring of tumor hypoxia is highly desirable for effective radiotherapy, and is the basis for improved treatment because hypoxic tumor cells are more resistant to radiation damage than fully oxygenated cells. We propose to use Cerenkov imaging to monitor tumor hypoxia by means of tissue blood oxygenation without the need for any exogenous contrast agent. Using a rodent hypoxia model, we demonstrate that Cerenkov imaging can be used as a noninvasive and noncontact method to measure tissue blood oxygenation level during radiation delivery. The data from Cerenkov imaging were validated using near infrared spectrometry methods. The results demonstrate the feasibility of using Cerenkov imaging to monitor tumor hypoxia during therapeutic radiation delivery. ( 2017 Optical Society of America

OCIS codes: (170.3880) Medical and biological imaging; (170.0170) Medical optics and biotechnology; (170.1470) Blood or tissue constituent monitoring; (170.3890) Medical optics instrumentation; (170.2655) Functional monitoring and imaging; (170.3660) Light propagation in tissues.

https://doi.org/10.1364/OL.42.003101

It has become increasingly evident that tumor hypoxia is heterogeneous and dynamic, commonly termed cyclic hypoxia, and that tumor hypoxia is linked to cancer prognosis in a highly complex manner [1]. In treating solid tumors, a fundamental goal is to deliver an optimal dose of radiation to eradicate cancer cells while minimizing damage to normal tissue. This is made more challenging because tumor hypoxia increases tumor cell resistance to radiotherapy [2]. With the availability of technologies for multidimensional radiation delivery, particularly the intensity-modulated radiation therapy (IMRT), highly targeted "dose sculpting" for tumor treatment becomes feasible. However, as tumor hypoxia can occur with periodicities ranging from minutes to hours and even to days, the ability of imaging tissue oxygenation at the time of dose delivery is critically important for improved outcomes of radiotherapy. A number of methods for tissue oxygenation imaging have been previously proposed using well-established medical imaging modalities, e.g., magnetic resonance imaging (MRI), positron emission tomography (PET), and single photon emission computed tomography (SPECT) [3-5]. However, applications of these methods have been limited during radiation delivery for a number of reasons: (1) challenges in system setup because of high equipment costs/ complexity, (2) patient safety concerns due to the use of radioactive agents, and (3) difficulties in quantitative interpretation as a result of the indirect and sometimes ambiguous relationship between the contrast mechanisms and the actual oxygenation level. Recently, detection of Cerenkov emission has been emerging as a promising approach to measure tissue hypoxia. Cerenkov emission refers to the emission of optical photons when a charged particle travels at a speed higher than the local phase velocity of light in the dielectric medium [6]. Cerenkov emission was observed in biological tissues when radioisotopes or radiotherapy beams were applied [7-10].

With its far-red and near infrared (NIR) components $(\sim 650-900 \mathrm{~nm})$, Cerenkov emission is well suited for deeptissue imaging because of the relatively low absorption and scattering within this spectral range. Tissue oxygenation state is directly correlated to the oxy- and deoxy-hemoglobin concentrations in blood, i.e., blood oxygen saturation $\left(\mathrm{StO}_{2}\right)$. Thanks to the characteristic wavelength-dependent absorption spectra of oxy-and deoxy-hemoglobin, as well as the noninvasive and noncontact capability of optical detection techniques, imaging of Cerenkov emission becomes a highly promising solution for deep-tissue $\mathrm{StO}_{2}$ measurement during radiation delivery. Recently, it has been reported that Cerenkov emission can be used to measure tissue $\mathrm{StO}_{2}$ via Cerenkov-excited phosphorescence [11] and spectral-fitted point-measurement [12]. In this Letter, we report a Cerenkov-based imaging method that noninvasively measures tissue $\mathrm{StO}_{2}$ during radiation delivery without using any exogenous contrast agents. This method measures a parameter (i.e., tissue absorption coefficient) that is 
directly linked to $\mathrm{StO}_{2}$, which determines tissue oxygenation $\left(p \mathrm{O}_{2}\right)$. Although highly complex, the absolute relationship between $\mathrm{StO}_{2}$ and tissue $p \mathrm{O}_{2}$ is deterministic and can be quantitatively measured with properly designed calibrations.

Our initial experiment in Cerenkov imaging was performed on an imaging phantom: excised chicken breast muscles. The source of high-energy radiation was a clinical linear accelerator (Novalis Tx, Varian Medical Systems) located at the Duke University Medical Center. Photon irradiation beams with energy levels of 6 and $15 \mathrm{MV}$ were used. The radiation doses were $1,5,10,20,50,100$, and $200 \mathrm{MU}$ (or monitor units) for each of the two energy levels at a dose rate of $600 \mathrm{MU} / \mathrm{min}$. The machine was calibrated to deliver $1 \mathrm{cGy} / \mathrm{MU}$ at a depth of $1.5 \mathrm{~cm}(6 \mathrm{MV})$ or $3 \mathrm{~cm}(15 \mathrm{MV})$ to a phantom located $100 \mathrm{~cm}$ away from the source. Images of Cerenkov emission were acquired using a back-illuminated charge-coupled device (CCD) camera (iKon-M934, Andor Technology), which was equipped with a general purpose imaging lens (Nikkor $50 \mathrm{~mm} f / 1.4$, Nikon). The CCD chip was cooled to $-85^{\circ} \mathrm{C}$ to reduce thermal noise from the photosensors. Optical acquisition time of the CCD was $20 \mathrm{~s}$ for all measurements. The photon irradiation beam was delivered vertically from the top. The CCD camera was positioned approximately $100 \mathrm{~cm}$ from the center of field of view (FOV) at an angle of $45^{\circ}$ from the irradiation beam. The imaging setup was enclosed in a dark imaging chamber that allowed the irradiation beam to pass without scattering or other secondary effects. In addition, all the light sources in the treatment room were turned off or blocked during optical acquisition. We observed that the intensity of Cerenkov emission (in terms of baseline-corrected CCD count) from the surface of the imaging phantom follows a linear relationship with respect to the delivered radiation dose, and that the intensity increases at a faster rate with respect to the dose at a higher energy (Fig. 1). The difference in signal intensity between the two energy levels was approximately $7 \%$ under our experimental conditions.

We developed a numeric model to estimate the relationship between tissue $\mathrm{StO}_{2}$ and Cerenkov emission. The spectrum of Cerenkov emission extends from ultraviolet (UV) to NIR following an inverse-square law with respect to emission wavelength in terms of the photon count [13]. In our numeric estimation, the spectral range of Cerenkov emission was set to $650-950 \mathrm{~nm}$, which was jointly limited by tissue attenuation and the profile of CCD sensitivity. The absorption coefficient

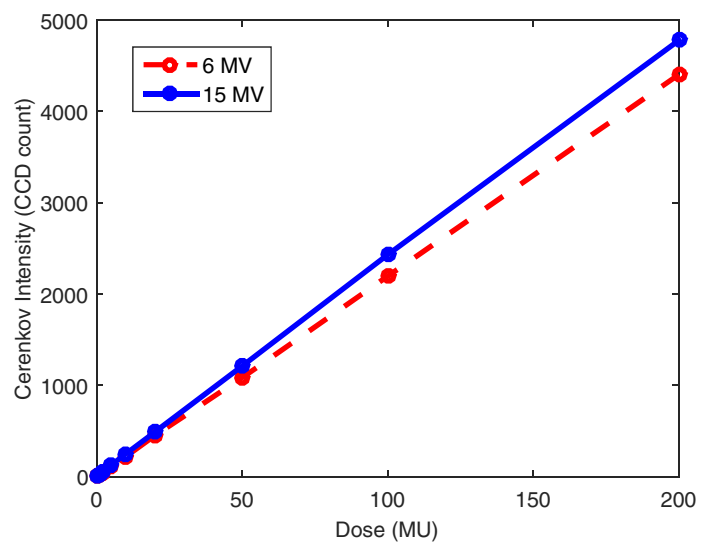

Fig. 1. Cerenkov signal intensity versus radiation dose at different energies in excised chicken breast muscles.

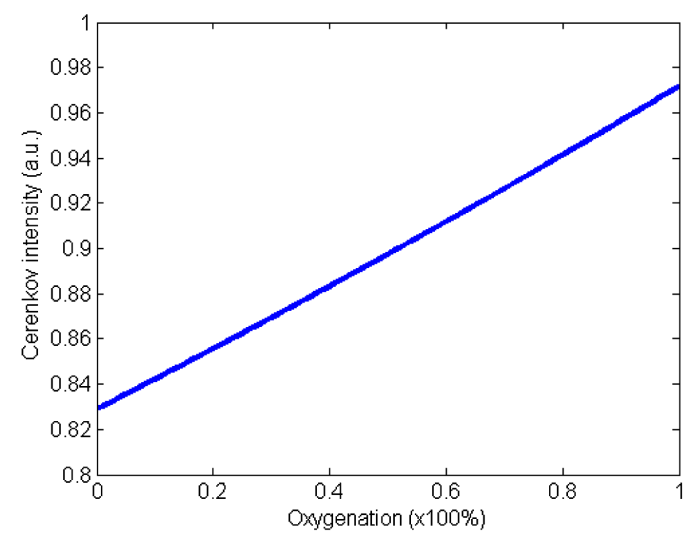

Fig. 2. Estimated dependency of Cerenkov signal on tissue $\mathrm{StO}_{2}$.

and the reduced scattering coefficient of biological tissue are wavelength-dependent, which can be described by a set of semiempirical equations [14]. Using our numeric model, we found that the intensity of Cerenkov emission increased almost linearly with respect to $\mathrm{StO}_{2}$, which is shown in Fig. 2 as a normalized curve of this dependency. The simulated Cerenkov intensity changed $\sim 14 \%$ over the entire range of $\mathrm{StO}_{2}$.

Our initial experiment and numerical estimation have lead us to hypothesize that Cerenkov imaging can measure tissue $\mathrm{StO}_{2}$ level in vivo. To test this hypothesis, we conducted two preliminary animal experiments. In these experiments, we used Cerenkov imaging to measure tissue $\mathrm{StO}_{2}$ level of the leg muscles of healthy adult rats (Sprague Dawley, Charles River Laboratories). Tissue $\mathrm{StO}_{2}$ was modulated by modifying the $\mathrm{O}_{2}$ fraction of breathing air for the animals under general anesthesia. The protocols for animal experiments were approved by the Duke University Institutional Animal Care and Use Committee (IACUC).

Hair on the thighs was removed using depilatory cream (Nair) $24 \mathrm{~h}$ prior to imaging. The animals were anesthetized with ketamine/xylazine $(60 / 6 \mathrm{mg} / \mathrm{kg}$ initial dose, intraperitoneal) and supplied with an $\mathrm{O}_{2}-\mathrm{N}_{2}$ mixture as breathing air via a nose cone. Oxygen fraction of the breathing air was gradually reduced from $100 \%$ (i.e., pure $\mathrm{O}_{2}$ ) to $0 \%$ (i.e., pure $\mathrm{N}_{2}$ ) at intermediate steps of $50,25,20,15$, and $10 \%$. At each step, the animals breathed the air- $\mathrm{N}_{2}$ mixture for 5-10 min to ensure the $\mathrm{StO}_{2}$ level was stabilized before imaging. The animals were euthanized by injection of a lethal dose of Euthasol before reaching the endpoint.

High-energy photon irradiation beams of $6 \mathrm{MV}$ were delivered to the right thighs with the same parameters as described in the phantom experiment: i.e., a radiation dose of $200 \mathrm{MU}$ with a dose rate of $600 \mathrm{MU} / \mathrm{min}$. The size of the irradiation beam was $2 \times 2 \mathrm{~cm}$. The distribution of radiation dose is highly uniform in the transverse direction and has a depth-dependent profile in the longitudinal direction subject to the specific anatomical structures. The variability of irradiation energy and dose were minimized by the routine quality assurance protocols for the clinical linear accelerator and were negligible for our imaging experiments.

For optical acquisition, the same instruments and configurations with similar parameters as detailed in the phantom experiment were also used: i.e., CCD cooled to $-85^{\circ} \mathrm{C}, 50 \mathrm{~mm}$ $f / 1.4$ lens, $100 \mathrm{~cm}$ lens-object distance, $45^{\circ}$ imaging angle, and 25 s acquisition time. A "dark" CCD image was acquired 
prior to the delivery of radiation to determine the CCD background signals and the readout noise in Cerenkov image acquisitions. The regions of interest (ROIs) for data analysis of all Cerenkov images were $0.4 \times 0.4 \mathrm{~cm}^{2}$.

In the first animal experiment (where $N=2$ ), we used a standard rodent oximeter (MouseOx Plus, Starr Life Sciences) to validate the measurements from the proposed Cerenkov imaging method. This oximeter has a specially designed collar to affix fiber optics onto the rodent's neck and measures the arterial $\mathrm{StO}_{2}$. It was disabled during Cerenkov imaging to avoid optical interference.

As shown in Fig. 3(a), the photon irradiation beam was aimed at the right thigh of one of the animals: the bright area marks the extent of irradiation impinging on the animal, and the crosshair indicates the FOV center of the irradiation beam. A raw Cerenkov image of the same animal is shown in Fig. 3(b). The random background noise in the image was due to scattered radiation in the treatment room, which was eliminated using a moving-average digital filter during the data processing stage. The intensity of Cerenkov signals (in terms of the CCD count) was plotted against the arterial hemoglobin saturation in Fig. 3(c): using least-squares linear regression, the regression " $R$ " values of the two data sets were 0.8 and 0.6 for Animals \#1 and \#2, respectively; the standard errors of all data points were 9.210.2 counts, which were fairly small $(\sim 4 \%)$ compared to their absolute intensities. The quantum efficiency profile of the CCD is shown in Fig. 3(d). As the spectral sensitivity profile of the CCD ( 400-900 nm at 50\%) overlaps with the NIR window of $\sim 650-900 \mathrm{~nm}$, the spectrum of acquired Cerenkov images is predominantly NIR. The results from the first experiment were consistent with our numerical estimation. However, it is noteworthy that the effects of physiological parameters on $\mathrm{StO}_{2}$ measurements were difficult to quantify in this experiment: e.g., arterial $\mathrm{StO}_{2}$ was known to be highly unstable under hypoxic conditions; $\mathrm{StO}_{2}$ in the arteries was not perfectly correlated with that in the leg muscles [15]; and the total blood volume may have changed due to induced hypoxia.

To address the limitations arising from the uncertain effects of the above physiological parameters, we designed and performed another experiment, in which all other main experimental conditions remained the same as the first one, except that the tissue $\mathrm{StO}_{2}$ level was measured locally on the leg muscles (as opposed to being measured globally at the carotid arteries). The instrument for local measurement of tissue $\mathrm{StO}_{2}$ was a commercially available visible/NIR spectral oximeter (Zenalux Biomedical) equipped with a handheld multifiber optical probe that can be arbitrarily positioned for superficial measurement.

During the experiment, a circular mark was drawn on the animal skin to ensure the same sampling locations for both Cerenkov and validation measurements. The validation measurements were taken immediately before and after each of the Cerenkov image acquisitions to minimize the effects of physiological fluctuations. The ROIs for data processing were centered at the circular marks drawn during the experiment.

Data from the second animal experiment (where $N=6$ ) are shown in Fig. 4. In Fig. 4(a), the mean pixel intensities within the specified ROIs of Cerenkov images were plotted against the $\mathrm{O}_{2}$ fraction of breathing air. In Fig. 4(b), tissue $\mathrm{StO}_{2}$ measured using the Zenalux oximeter at the same ROIs was plotted similarly.
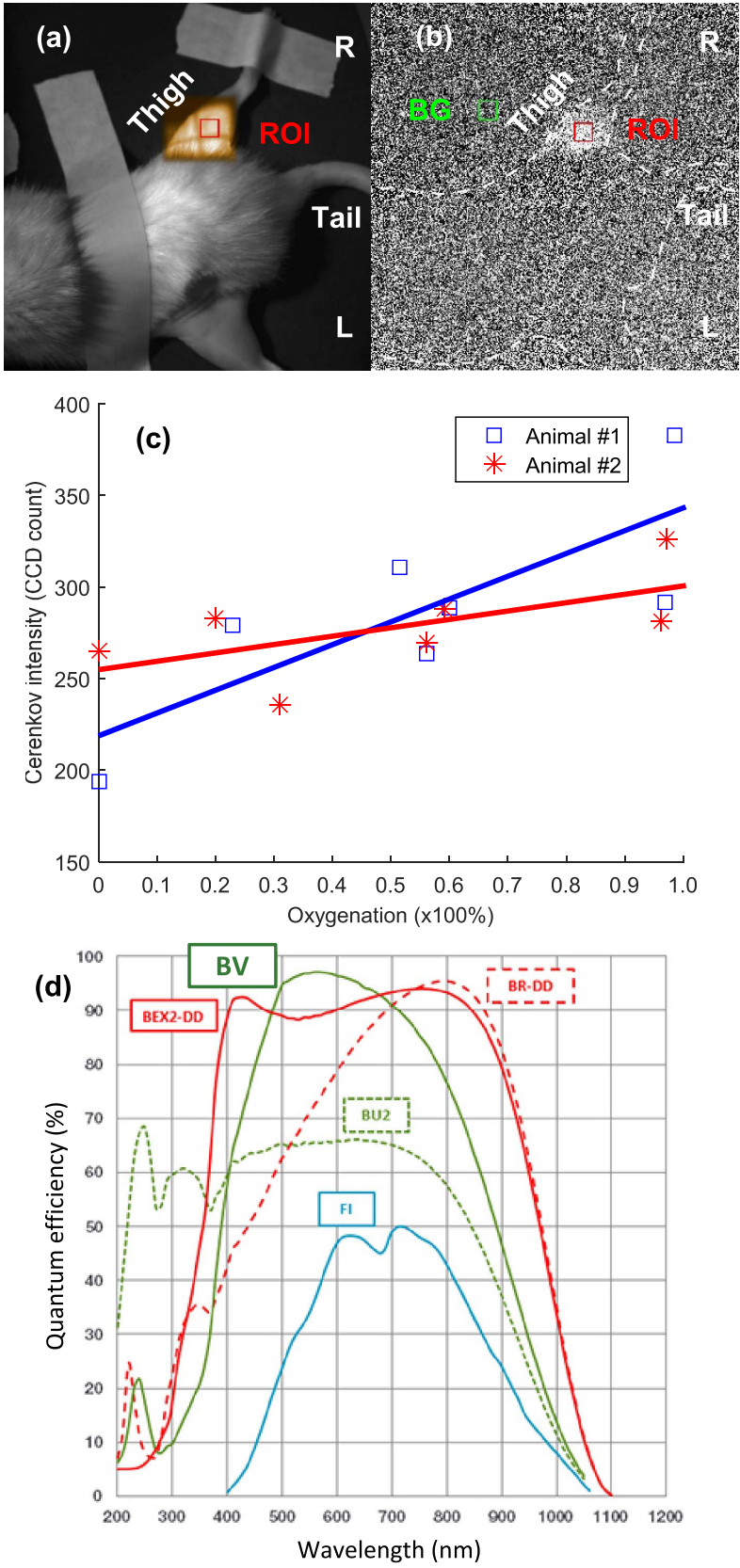

Fig. 3. Animal experiment \#1: (a) irradiation beam marked by the linear accelerator crosshair light superimposed with the animal outline (white dashed lines; indicating the right leg of animal, pointed upward toward the 12 o'clock position) and the ROI for Cerenkov signal intensity (red rectangle); (b) Cerenkov image of an animal breathing $100 \% \mathrm{O}_{2}$ under $200 \mathrm{MU}$ radiation superimposed with the same animal outline, ROI, and background ("BG," green rectangle); (c) in vivo Cerenkov intensity as a function of hemoglobin saturation in the carotid artery; and (d) spectral quantum efficiency of the back-illuminated ("BV") CCD (adopted from product specifications sheet of Andor Technology).

Both data sets from Cerenkov imaging and NIR oximetry showed consistent patterns that we have expected: the tissue $\mathrm{StO}_{2}$ level remained at a constantly high level under hyperoxia (i.e., $\mathrm{O}_{2}$ fraction $>50 \%$ ), decreased moderately when the animals 

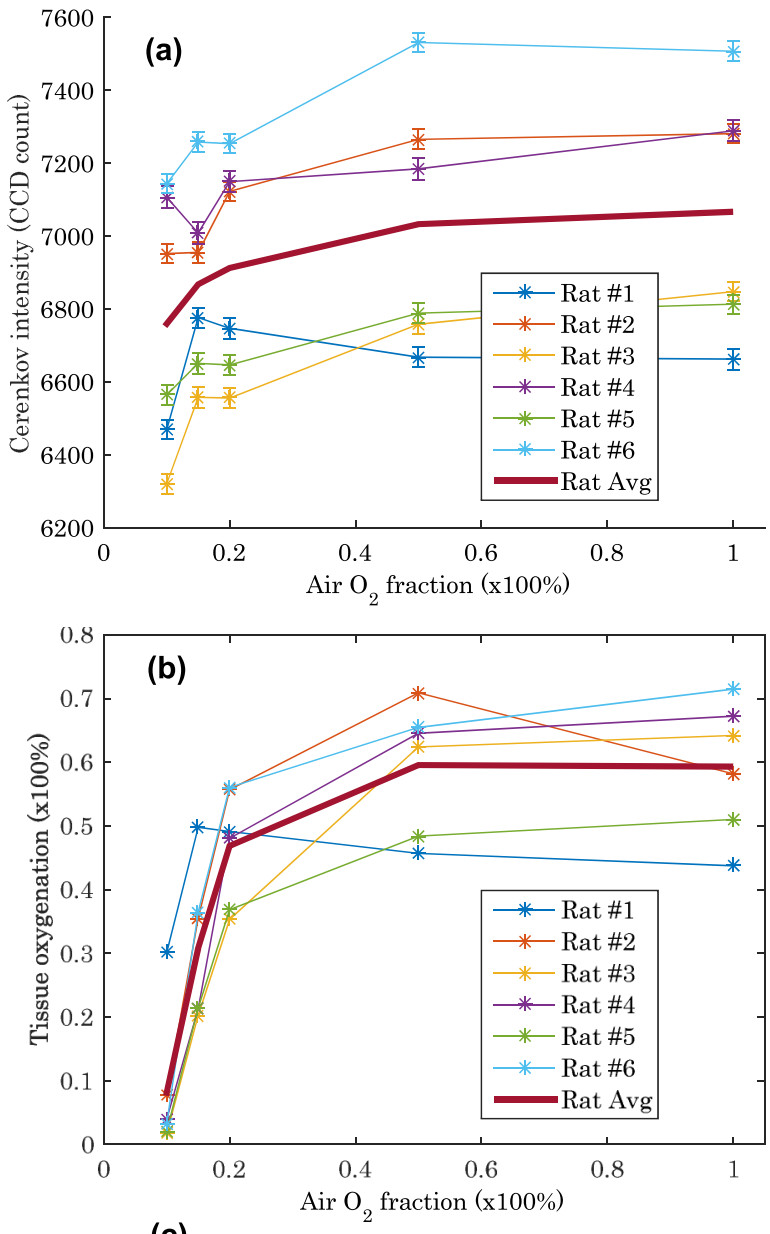

(c)

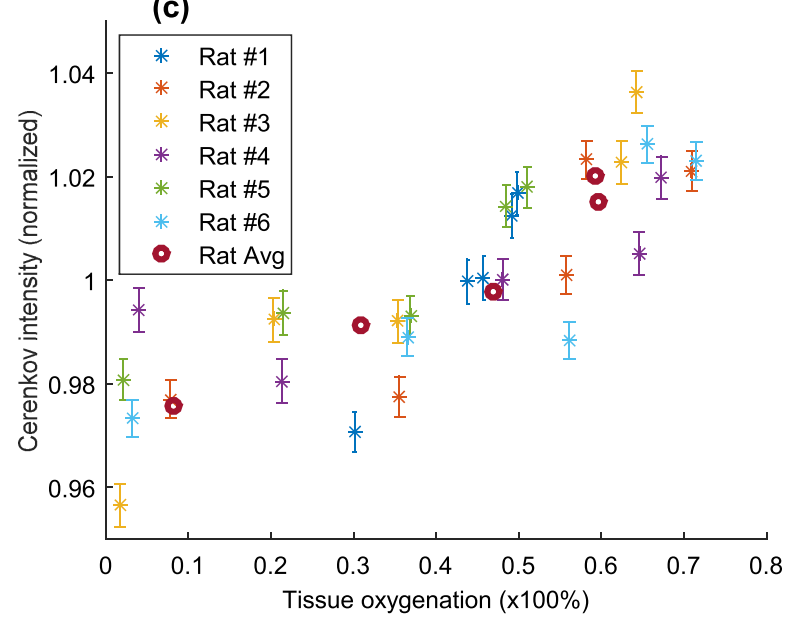

Fig. 4. Animal experiment \#2: (a) tissue $\mathrm{StO}_{2}$ measured using the proposed Cerenkov imaging method, (b) validation measurement at the same ROIs on the animals using Zenalux oximeter, and (c) normalized Cerenkov data versus Zenalux tissue $\mathrm{StO}_{2}$ data.

were transitioning from hyperoxia to normoxia $\left(\mathrm{O}_{2} 20-50 \%\right)$, and declined dramatically under hypoxic conditions $\left(\mathrm{O}_{2}<20 \%\right)$.

Although individual animals may respond differently to the same oxygen challenge, the data from each individual showed highly consistent patterns in Cerenkov and Zenalux data. To further depict the relationship between the two measurements, the Cerenkov signals were plotted against the tissue $\mathrm{StO}_{2}$ data obtained from the Zenalux data [Fig. 4(c)]. The Cerenkov signals were normalized by the average values of individual animals to reduce the effects of variations of image acquisition angles and the physiological responses to the changes in $\mathrm{O}_{2}$ fraction of the breathing air. In the averaged data, the Cerenkov intensity changed $\sim 5 \%$ over the $\mathrm{StO}_{2}$ range of $10-60 \%$, which is consistent with our numerical estimation in the same range ( $\sim 6 \%$ change, Fig. 2$)$. We note that a number of important system and physiological parameters are needed to quantitatively model Cerenkov emission. In practice, however, these parameters are typically constant within the time frame of Cerenkov imaging. As a result, quantitative measurement may be achieved with proper calibration methods, which is a direction of our future work.

We conclude that Cerenkov imaging can be used as a noninvasive and noncontact method to measure in vivo tissue $\mathrm{StO}_{2}$ level during the delivery of radiation dose, and that this method has been qualitatively validated with a rat hypoxia model using NIR spectrometry. More importantly, no exogenous contrast agent was used in the proposed Cerenkov imaging method, which opens great potentials for many exciting preclinical and clinical applications.

Funding. Duke University School of Medicine (Duke Department of Radiology Putman Seed Fund 2016-01); Duke University (Duke Cancer Institute C3O Pilot 2017).

Acknowledgment. G. M. P. and Duke University have a financial interest in Zenalux Biomedical Inc. and the underlying technology that was used as a tool in this work. The authors also acknowledge the use of the Preclinical Translational Research Unit in conducting this research.

\section{REFERENCES}

1. M. W. Dewhirst, Radiat. Res. 172, 653 (2009).

2. L. H. Gray, A. D. Conger, M. Ebert, S. Hornsey, and O. C. A. Scott, Br. J. Radiol. 26, 638 (1953).

3. C. Baudelet, R. Ansiaux, B. F. Jordan, X. Havaux, B. Macq, and B. Gallez, Phys. Med. Biol. 49, 3389 (2004).

4. N. Lee, S. Nehmeh, H. Schoder, M. Fury, K. Chan, C. C. Ling, and J. Humm, Int. J. Radiat. Oncol. Biol. Phys. 75, 101 (2009).

5. C. J. Koch and S. M. Evans, Adv. Exp. Med. Biol. 510, 285 (2003).

6. P. Cerenkov, C. R. Acad. Sci. URSS 3, 451 (1934).

7. R. Robertson, M. S. Germanos, C. Li, G. S. Mitchell, S. R. Cherry, and M. D. Silva, Phys. Med. Biol. 54, N355 (2009).

8. J. Axelsson, S. C. Davis, D. J. Gladstone, and B. W. Pogue, Med. Phys. 38, 4127 (2011).

9. A. E. Spinelli, M. Ferdeghini, C. Cavedon, E. Zivelonghi, R Calandrino, A. Fenzi, A. Sbarbati, and F. Boschi, J. Biomed. Opt. 18, 20502 (2013).

10. X. Zhang, C. Kuo, A. Moore, and C. Ran, PLoS ONE 8, e62007 (2013).

11. R. Zhang, S. C. Davis, J. L. Demers, A. K. Glaser, D. J. Gladstone, T. V. Esipova, S. A. Vinogradov, and B. W. Pogue, J. Biomed. Opt. 18, 50503 (2013).

12. J. Axelsson, A. K. Glaser, D. J. Gladstone, and B. W. Pogue, Opt. Express 20, 5133 (2012).

13. J. V. Jelley, Br. J. Appl. Phys. 6, 227 (1955).

14. G. Alexandrakis, F. R. Rannou, and A. F. Chatziioannou, Phys. Med. Biol. 50, 4225 (2005).

15. L. I. Cardenas-Navia, D. Yu, R. D. Braun, D. M. Brizel, T. W. Secomb, and M. W. Dewhirst, Cancer Res. 64, 6010 (2004). 